\title{
A Fixed Ratio Combination of Insulin Degludec and Liraglutide (IDegLira) Reduces Glycemic Fluctuation and Brings More Patients with Type 2 Diabetes Within Blood Glucose Target Ranges
}

\author{
Allen B. King, MD, ${ }^{1}$ Athena Philis-Tsimikas, $\mathrm{MD}^{2}$, Eric S. Kilpatrick, MBChB, MD, \\ Irene H. Langbakke, MD, Kamilla Begtrup, MSc, and Tina Vilsbøll, MD, DMSc ${ }^{4}$
}

\begin{abstract}
Background: Reducing glycemic fluctuation is important for optimal diabetes management. This post hoc analysis examined glycemic fluctuations and the proportion of subjects achieving recommended blood glucose targets with the fixed ratio combination of insulin degludec and liraglutide (IDegLira) compared to insulin degludec (IDeg) and liraglutide alone.

Methods: We analyzed nine-point self-monitored blood glucose (SMBG) profiles from two randomized trials involving IDegLira in patients with type 2 diabetes (T2D), and continuous glucose monitoring (CGM) data from a subset of patients in one of these trials to assess glycemic fluctuation and day-to-day variability.

Results: Compared with IDeg, IDegLira resulted in a greater proportion of subjects with SMBG values within target ranges (3.9-9.0 mmol/L) than IDeg for all pre- and postprandial values, and for the full nine-point profile $(P<0.05$ for all). IDegLira also resulted in a greater reduction in the range of SMBG values over $24 \mathrm{~h}$ than IDeg $(P \leq 0.0001)$. CGM data showed that IDegLira provided greater reductions in interstitial glucose (IG) fluctuation $(P=0.0018)$ and postprandial IG increment $(P=0.0288)$ compared with IDeg. Compared with liraglutide, IDegLira brought a higher proportion of subjects within SMBG target ranges (all pre- and all postprandial values, and the full nine-point profile, $P<0.01$ for all) and resulted in a greater reduction of time outside the IG target range $(P=0.0072)$. IDegLira also reduced mean IG more than liraglutide $(P<0.0001)$.

Conclusions: Treatment with IDegLira allows more patients with T2D to maintain blood glucose within target ranges throughout the day than either IDeg or liraglutide alone.
\end{abstract}

Keywords: Insulin degludec, Liraglutide, Type 2 diabetes, GLP-1 analog, Continuous glucose monitoring.

\section{Introduction}

$\mathbf{T}$ HERAPEUTIC AIMS IN DIABETES include achieving target glycated hemoglobin $\left(\mathrm{HbA}_{1 \mathrm{c}}\right)$ levels and reducing glycemic fluctuations to achieve a balance between glycemic control and avoidance of hypoglycemia. ${ }^{1,2}$ Unfortunately, patients with an acceptable $\mathrm{HbA}_{1 \mathrm{c}}$ level may still experience significant glycemic fluctuation over a 24 -h period. ${ }^{3}$ There is evidence that glycemic fluctuation is an independent risk factor for diabetic complications, ${ }^{4,5}$ predictive of hypoglycemia, ${ }^{6}$ and when manifesting as postprandial hyperglycemia may contribute to vascular complications. ${ }^{7-9}$ Despite some

\footnotetext{
${ }^{1}$ Diabetes Care Center, Salinas, California.

${ }^{2}$ Scripps Whittier Diabetes Institute, San Diego, California.

${ }^{3}$ Sidra Medical and Research Center, Doha, Qatar.

${ }^{4}$ Novo Nordisk A/S, Søborg, Denmark.

${ }^{5}$ Center for Diabetes Research, Gentofte Hospital, University of Copenhagen, Copenhagen, Denmark
}

(C) Allen B. King et al., 2017; Published by Mary Ann Liebert, Inc. This Open Access article is distributed under the terms of the Creative Commons Attribution Noncommercial License (http://creativecommons.org/licenses/by-nc/4.0/) which permits any noncommercial use, distribution, and reproduction in any medium, provided the original author(s) and the source are credited. 
controversy on these issues, ${ }^{10,11}$ reducing glycemic fluctuation is important for optimal glycemic control, ${ }^{4}$ and treatments that offer reductions in both $\mathrm{HbA}_{1 \mathrm{c}}$ and glycemic fluctuation are desirable.

IDegLira, a fixed ratio combination of insulin degludec (IDeg), a long-acting basal insulin, and the glucagon-like peptide-1 (GLP-1) receptor agonist, liraglutide, has demonstrated significantly greater reductions in $\mathrm{HbA}_{1 \mathrm{c}}$ compared to the individual components liraglutide and IDeg alone. ${ }^{12-15}$ IDegLira has also been shown to have favorable effects on body weight and reduced risk of hypoglycemia compared to IDeg alone. ${ }^{12-15}$ These observations are likely explained by the complementary mode of action of the two components of IDegLira, namely the ability of IDeg to reduce fasting plasma glucose $^{16}$ and the glucose-dependent effects of liraglutide, both lowers fasting plasma glucose and reduces postprandial glucose excursions. ${ }^{17}$

The objective of this post hoc analysis was to use selfmonitored blood glucose (SMBG) and continuous glucose monitoring (CGM) to determine whether or not IDegLira would bring more patients with type 2 diabetes (T2D) within blood glucose target ranges, compared to its individual components. In this article, we describe glycemic fluctuation from a patient's average blood glucose values and explore day-today variability by comparing the standard deviation (SD) of consecutive blood glucose concentrations.

\section{Materials and Methods}

\section{DUAL I extension and DUAL // study overviews}

This post hoc analysis used data from the DUAL I extension and DUAL II trials in patients with T2D; detailed trial designs and methods were reported previously. ${ }^{13-15}$ Briefly, DUAL I was a 26 -week trial ${ }^{14}$ with an extension to 52 weeks ${ }^{15}$ comparing IDegLira with IDeg or liraglutide in insulin-naïve patients uncontrolled on oral antidiabetic drugs (OADs; in this case being metformin with or without pioglitazone). Throughout this article, when referring to DUAL I, results from the full 52-week trial are reported. In DUAL II, IDegLira was compared with IDeg for 26 weeks in patients who were previously uncontrolled on 20 to $40 \mathrm{U}$ of basal insulin plus metformin, with or without sulfonylureas or glinides; these latter two classes were discontinued at randomization. ${ }^{13}$ In DUAL II, IDeg was limited to a maximum dose of $50 \mathrm{U}$ so that the contribution of the liraglutide component of IDegLira could be evaluated at equivalent insulin doses. The trial protocols were approved by independent ethics committees or institutional review boards at all participating institutions and conducted in accordance with the Declaration of Helsinki and Good Clinical Practice guidelines. ${ }^{18,19}$ Written informed consent from all patients was obtained before enrollment. The baseline characteristics of the patients in the two trials are summarized in the Supplementary Tables S1 and S2 (Supplementary Data are available online at www.liebertpub.com/dia).

\section{SMBG profiles}

SMBG levels were assessed in the DUAL I extension and DUAL II trials using hand-held glucose meters (Abbott Diabetes Care, Alameda) calibrated to display plasma equivalent values of capillary whole blood glucose measurements. SMBG levels were recorded in the diary in the following se- quence of time points: (1) just before breakfast; (2) $90 \mathrm{~min}$ after the start of breakfast; (3) just before lunch; (4) $90 \mathrm{~min}$ after the start of lunch; (5) just before dinner; (6) 90 min after the start of dinner; (7) at bedtime; (8) at 4 am; and (9) just before breakfast the following day. These nine-point profiles were completed within 1 week before site visits on a day when the subject did not anticipate unusual strenuous exercise. The 90-min postprandial interval was selected as the mid-point in the $1-2 \mathrm{~h}$ timeframe recommended for assessing postprandial plasma glucose targets, and in an effort to provide a closer reflection of postprandial peak than the common 2-h postprandial interval. $^{4,20}$ In addition, fasting prebreakfast SMBG was measured daily for both IDeg and IDegLira treated patients (the two study arms that included dose titration), and 3 days a week for liraglutide-treated patients (as per study protocol).

Interpreting nine-point SMBG profiles. We assessed the proportions of patients treated with IDegLira, IDeg, or liraglutide, who achieved SMBG values (plasma equivalent values) from the nine-point profile within recommended capillary plasma glucose target ranges at baseline and end-of-trial (EOT). ${ }^{4,21}$ The preprandial (before breakfast, lunch, dinner, and breakfast the following day) target was achieved if SMBG was $\geq 3.9$ and $\leq 7.2 \mathrm{mmol} / \mathrm{L}$ at all four assessments. ${ }^{21}$ The postprandial (90 min after all three meals) target was SMBG $<9.0 \mathrm{mmol} / \mathrm{L}$ at all three assessments. ${ }^{4}$ Patients were considered to have the entire nine-point profile within target range if SMBG was $\geq 3.9$ and $<9.0 \mathrm{mmol} / \mathrm{L}$ at all nine assessments (all pre- and postprandial time points, before bedtime and $4 \mathrm{am}$ ).

We also assessed the range of SMBG values in the ninepoint profile (the difference between the minimum and maximum SMBG values recorded) for each individual patient, at baseline and at EOT. The difference in range from baseline to EOT was determined for each patient, and the change in range was compared across treatments arms.

Analysis of parameters determined from the nine-point profiles was based on the full analysis set (FAS; all randomized subjects), with the last observation carried forward (LOCF) method of imputation for all subjects with a full nine-point profile at baseline. Continuous endpoints were analyzed using an analysis of covariance (ANCOVA) model with treatment, region, sub-study, baseline $\mathrm{HbA}_{1 \mathrm{c}}$ stratum $(\leq 8.3 \%$ [ $\leq 67 \mathrm{mmol} / \mathrm{mol}],>8.3 \%$ [ $>67 \mathrm{mmol} / \mathrm{mol}])$, and previous OAD treatment as fixed effects, and the baseline value of the parameter included as a covariate. Binary endpoints were analyzed using logistic regression with treatment, region, baseline $\mathrm{HbA}_{1 \mathrm{c}}$ stratum $(\leq 8.3 \% \quad[\leq 67 \mathrm{mmol} / \mathrm{mol}$, $>8.3 \%$ [ $>67 \mathrm{mmol} / \mathrm{mol}]$ ), and previous OAD treatment as fixed effects.

Day-to-day variability with prebreakfast SMBG values. The coefficient of variation (CV, defined as the SD of the measurements as a percentage of the mean) of prebreakfast SMBG was calculated for each patient during the maintenance period of treatment to assess day-to-day variability. The maintenance period was defined as week 16 (inclusive) onward, and covered 38 visits over 37 weeks in DUAL I (weeks 16-52; includes regular and follow-up visits in week 27), and 11 visits over 11 weeks in DUAL II (weeks 16-26).

The CV of the individual prebreakfast SMBG values during the maintenance period was log-transformed before 
analysis. Data were analyzed using an ANCOVA model with treatment, region, sub-study, baseline $\mathrm{HbA}_{1 \mathrm{c}}$ stratum $(\leq 8.3 \%$ [ $\leq 67 \mathrm{mmol} / \mathrm{mol}],>8.3 \%$ [ $>67 \mathrm{mmol} / \mathrm{mol}])$, and previous $\mathrm{OAD}$ treatment as fixed effects.

\section{Continuous glucose monitoring}

CGM was used to characterize interstitial glucose (IG) excursions over a 24 -h period encompassing all three main meals, at baseline and at week 52, in the DUAL I extension sub-study of 260 patients. Subjects wore the CGM device (iPro1 and iPro2 [Europe and Australia], Medtronic International, Tolochenaz, Switzerland) for a minimum of $72 \mathrm{~h}$ for each recording, performed 3-4 days immediately before site visits. IG measurements were recorded every $5 \mathrm{~min}$ during each 72-h period and calibration of CGM devices was completed, using SMBG values (methods described previously), four times per day as per the manufacturer's instructions. Subjects were instructed to maintain diet, OAD dose, and investigational product treatment dose during the 72-h period. Subjects recorded meal times and dates in diaries during CGM; meal times were confirmed by cross-checking against rises in IG on the CGM profile. Successfully uploaded CGM data were blind-reviewed and quality checked for missing information, such as meal times. CGM data were not collected in the DUAL II study.

We assessed the following CGM parameters: mean IG; IG fluctuation (adjusted integrated absolute distance from the mean profile, that is, flatness of IG profile; Formula S1); mean amplitude of glycemic excursions (MAGE); low blood glucose index (LBGI); high blood glucose index (HBGI); postprandial IG increment across all meals (90 min after the start of each meal-in line with the postprandial time point used for the nine-point SMBG profiles); time spent with $\mathrm{IG}>3.9 \mathrm{mmol} /$ $\mathrm{L}$ and $\leq 9.0 \mathrm{mmol} / \mathrm{L}$, as a measure of the time that glucose profile was within range; duration and episodes (number of times the profile goes below the threshold after having been either above or missing) of $\mathrm{IG}<3.1$ and $<3.9 \mathrm{mmol} / \mathrm{L}$, including during the nocturnal period (00:01-05:59); day-to-day IG variability (SD of daily [24 h] mean IG); and day-to-day variability of fasting IG (SD of daily fasting IG, 20 min before breakfast).

Objective decision rules were used to handle incomplete CGM data. If there was no valid sensor value $5 \mathrm{~min}$ before, or 85 to $95 \mathrm{~min}$ after, the start of a meal, the meal increment was considered missing. If this total interval included a $30 \mathrm{~min}$ continuous period without sensor values, the meal was considered missing. For incomplete profiles, mean IG and IG fluctuation were derived from periods with available data.

All CGM parameters, except for episodes of IG $<3.1$ and $<3.9 \mathrm{mmol} / \mathrm{L}$, were analyzed based on observed data using an ANCOVA model with treatment, region, baseline $\mathrm{HbA}_{1 \mathrm{c}}$ stratum $(\leq 8.3 \%$ [ $\leq 67 \mathrm{mmol} / \mathrm{mol}],>8.3 \%[>67 \mathrm{mmol} / \mathrm{mol}])$; and previous OAD treatment as fixed effects, and baseline values of parameters included as covariates for mean IG and postprandial IG increment. The SD of daily mean and daily fasting IG was log-transformed before analysis. Analysis of the episodes of $\mathrm{IG}<3.1 \mathrm{mmol} / \mathrm{L}$ and $<3.9 \mathrm{mmol} / \mathrm{L}$ was based on observed data using a negative binomial regression model with a log link and the logarithm of the profile duration as offset, and treatment, region, baseline $\mathrm{HbA}_{1 \mathrm{c}}$ stratum $(\leq 8.3 \%$ [ $\leq 67 \mathrm{mmol} / \mathrm{mol}],>8.3 \% \quad[>67 \mathrm{mmol} / \mathrm{mol}])$ and previous OAD treatment as fixed effects.

\section{Results}

As described previously, baseline characteristics were well matched across treatment groups in the DUAL I extension (Supplementary Table S1) ${ }^{15}$ and DUAL II (Supplementary Table S2) trials. ${ }^{13}$

\section{SMBG profiles}

Nine-point SMBG profiles. Nine-point SMBG profiles were analyzed for all subjects with a full profile at baseline: $90 \%$ of subjects (FAS) in DUAL I extension ${ }^{15}$ and $95 \%$ in DUAL II, ${ }^{13}$ respectively.

At baseline, the proportion of patients with SMBG values (plasma equivalent values) within the target ranges was similar across treatments in the two trials (2\%-7\%, Table 1). At EOT, the proportion of patients with all three postprandial SMBG measurements $<9 \mathrm{mmol} / \mathrm{L}$ was greater with IDegLira versus IDeg (odds ratio [OR] 1.77, 95\% confidence interval [CI] 1.37 to $2.28, P<0.0001$ ) or liraglutide (OR $1.93,95 \%$ CI 1.49 to $2.49, P<0.0001)$ alone in DUAL I extension and versus IDeg in DUAL II (OR 1.90, 95\% CI 1.17 to 3.07, $P=0.0093$ ) (Table 1 and Supplementary Figs. S1 and S2; Supplementary Data are available online at www.liebertpub. $\mathrm{com} / \mathrm{dia}$ ). The proportion of patients with all four preprandial SMBG measurements within target range (3.9-7.2 mmol/L) was also greater with IDegLira versus IDeg (OR 1.34, 95\% CI 1.05 to $1.72, P=0.0204$ ) or liraglutide (OR $2.06,95 \% \mathrm{CI}$ 1.59 to $2.67, P<0.0001)$ alone in DUAL I extension and versus IDeg in DUAL II (OR 2.29, 95\% CI 1.41 to $3.73, P=$ 0.0008) (Table 1 and Supplementary Figs. S3 and S4). Similarly, the proportion of patients with all nine SMBG measurements within target range $(\geq 3.9$ and $<9 \mathrm{mmol} / \mathrm{L})$ was greater with IDegLira versus IDeg (OR 1.79, 95\% CI 1.36 to $2.36, P<0.0001$ ) or liraglutide (OR $1.46,95 \%$ CI 1.12 to 1.92 , $P=0.0059)$ alone in DUAL I extension and versus IDeg in DUAL II (OR 2.07, 95\% CI 1.22 to $3.49, P=0.0067$ ) (Table 1 and Supplementary Fig. S5).

The reduction in nine-point profile range (minimummaximum) from baseline to EOT was statistically significantly greater with IDegLira than with IDeg (DUAL I: estimated treatment difference [ETD] $-0.80 \mathrm{mmol} / \mathrm{L}, 95 \% \mathrm{CI}-1.08$ to $-0.52, P<0.0001$; DUAL II: ETD $-1.02 \mathrm{mmol} / \mathrm{L}, 95 \% \mathrm{CI}$ -1.53 to $-0.50, P=0.0001$ ), and was similar between IDegLira and liraglutide (ETD $0.01 \mathrm{mmol} / \mathrm{L}, 95 \% \mathrm{CI}-0.27$ to 0.30 , $P=$ NS) (Fig. 1).

Day-to-day variability with prebreakfast SMBG values. There was no statistically significant difference in CV of prebreakfast SMBG in the maintenance period in DUAL I extension between IDegLira and IDeg (treatment ratio: 0.95, 95\% CI 0.90 to $1.00, P=0.0638$ ), but $C V$ was statistically significantly greater with IDegLira compared with liraglutide (treatment ratio: $1.09,95 \% \mathrm{CI} 1.04$ to $1.15, P=0.0008$ ). In DUAL II, the $\mathrm{CV}$ of prebreakfast SMBG during the maintenance period was statistically significantly lower with IDegLira than with IDeg (treatment ratio: $0.88,95 \%$ CI 0.81 to $0.97, P=0.0091$ ).

\section{Continuous glucose monitoring}

Baseline characteristics of subjects in the CGM sub-study were well matched and similar to the full trial populations (Table 2 and Supplementary Table S1) ${ }^{14,15}$ Due to subject 


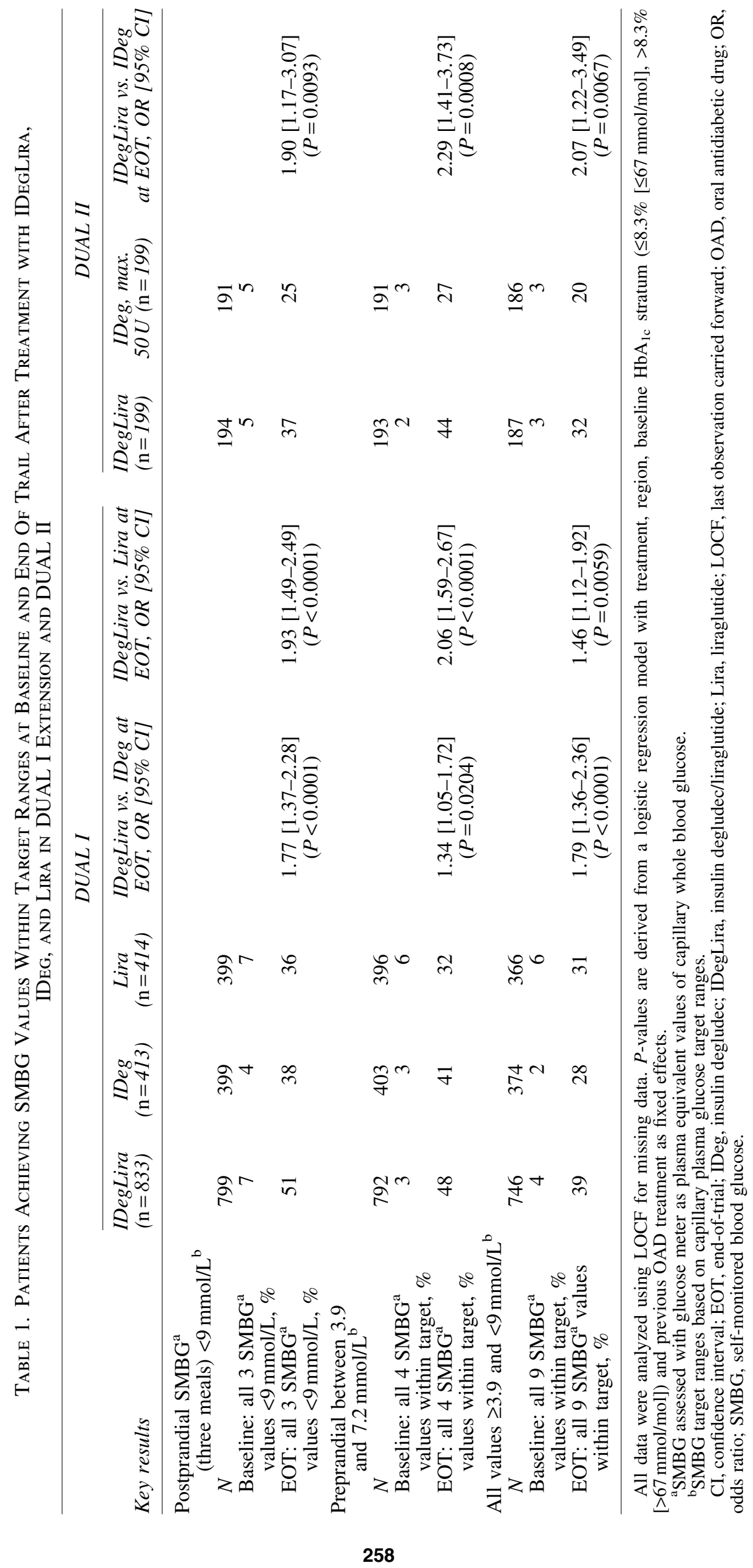


a FOR ILLUSTRATIVE PURPOSES ONLY‡

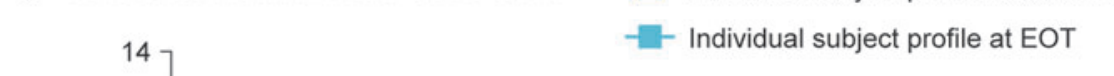

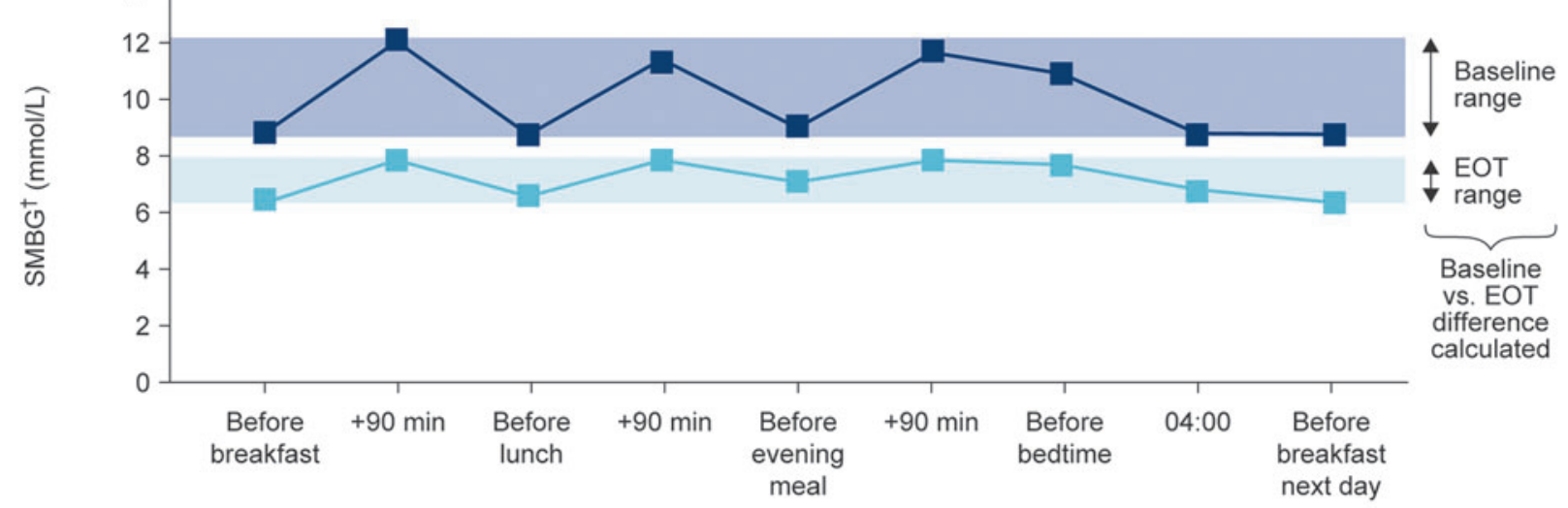

b

DUAL I extension

DUAL II
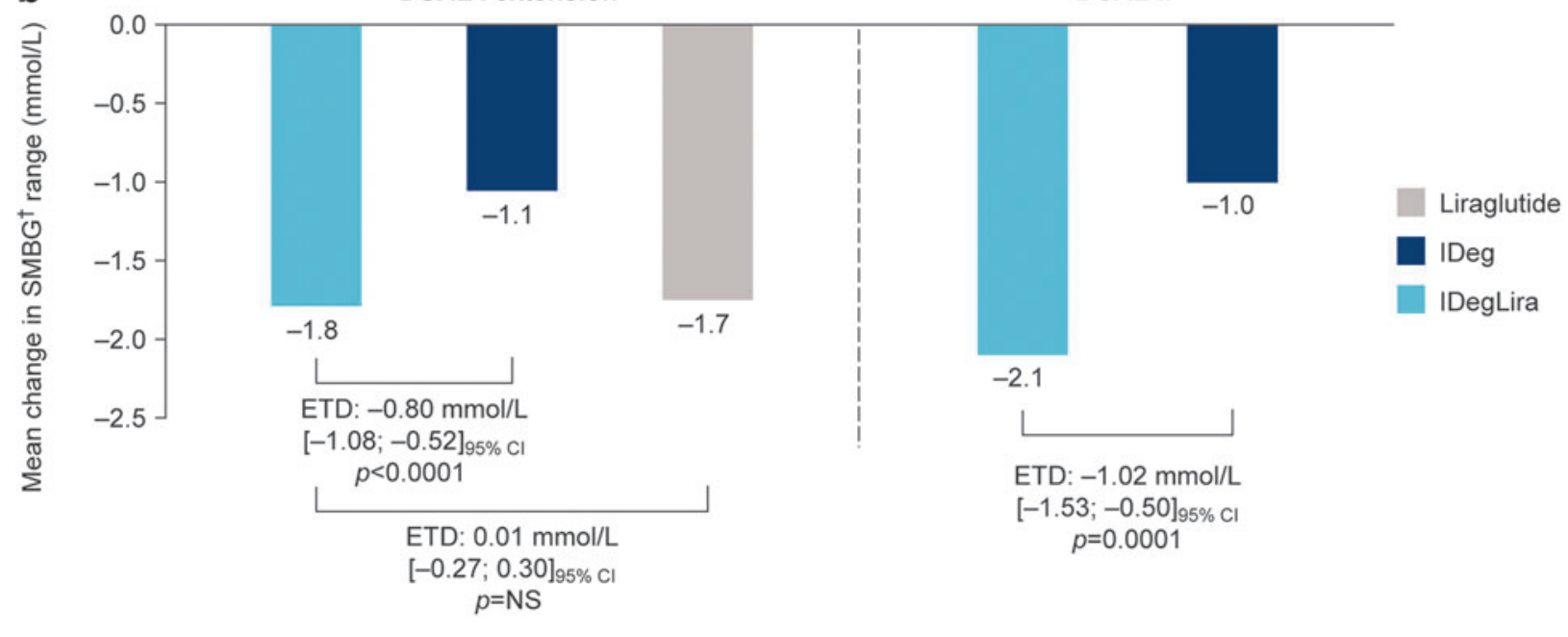

FIG. 1. Nine-point $\mathrm{SMBG}^{\dagger}$ profile ${ }^{\ddagger}$ showing calculation of range (a) and change from baseline to EOT in DUAL I and DUAL II (b). ${ }^{\dagger}$ SMBG assessed with glucose meter as plasma equivalent values of capillary whole blood glucose. ${ }^{\dagger}$ Illustrative example not intended to represent actual patients or treatment effects. Data are based on FAS, with LOCF for all subjects with a full nine-point profile at baseline; $P$-values are from ANCOVA with treatment, region, baseline $\mathrm{HbA}_{1 \mathrm{c}}$ stratum $(\leq 8.3 \%$ $[\leq 67 \mathrm{mmol} / \mathrm{mol}],>8.3 \%$ [ $>67 \mathrm{mmol} / \mathrm{mol}])$, and previous OAD treatment as fixed effects, and baseline value of the parameter included as covariate. ANCOVA, analysis of covariance; EOT, end-of-trial; ETD, estimated treatment difference; FAS, full analysis set; $\mathrm{HbA}_{1 \mathrm{c}}$, glycosylated hemoglobin; IDeg, insulin degludec; IDegLira, fixed ratio combination of insulin degludec and liraglutide; Lira, liraglutide; LOCF, last observation carried forward; OAD, oral antidiabetic drug; NS, not significant; SMBG, self-monitored blood glucose.

\section{Table 2. DuAl I Extension Continuous Glucose Monitoring Sub-Study Baseline Characteristics}

\begin{tabular}{lccc}
\hline Characteristic & IDegLira $(\mathrm{n}=131)$ & IDeg $(\mathrm{n}=64)$ & Liraglutide $(\mathrm{n}=65)$ \\
\hline Male/female, $\%$ & $55 / 45$ & $56 / 44$ & $46 / 54$ \\
Age, years & $54.4(9.3)$ & $55.0(8.5)$ & $55.0(10.3)$ \\
BMI, kg/m & $32.5(4.4)$ & $32.4(4.5)$ & $32.3(4.8)$ \\
Duration of diabetes, years & $7.5(5.7)$ & $7.5(4.9)$ & $8.1(5.1)$ \\
$\mathrm{HbA}_{1 \mathrm{c}}, \%$ & $8.2(0.9)$ & $8.2(0.9)$ & $8.3(1.0)$ \\
$\mathrm{HbA}_{1 \mathrm{c}}, \mathrm{mmol} / \mathrm{mol}^{\mathrm{a}}$ & $66(10)$ & $66(10)$ & $67(11)$ \\
FPG $^{2} \mathrm{mmol} / \mathrm{L}$ & $9.2(2.3)$ & $9.1(2.7)$ & $9.2(2.4)$ \\
Fasting C-peptide, nmol/L & $0.79(0.37)^{\mathrm{b}}$ & $0.85(0.48)^{\mathrm{c}}$ & $0.81(0.37)^{\mathrm{d}}$ \\
\hline
\end{tabular}

Values are mean (SD) unless otherwise stated.

${ }^{\mathrm{a} C}$ Calculated, not measured.

$\mathrm{b}_{n=127 .}$

${ }^{c} n=62$.

$\mathrm{d}_{n=63}$.

BMI, body mass index; CGM, continuous glucose monitoring; FPG, fasting plasma glucose; $\mathrm{HbA}_{1 \mathrm{c}}$, glycated hemoglobin; IDeg, insulin degludec; IDegLira, fixed-ratio combination of insulin degludec and liraglutide; SD, standard deviation. 
withdrawal, site closure, and technical issues (primarily difficulties with insertion of the sensor and sites' failure to activate the devices), usable CGM data were not available from a number of subjects (Supplementary Table S3). CGM profiles were therefore missing from $20 \%$ of subjects at baseline, and $38 \%$ at week 52; profiles were missing from $13 \%$ of subjects at both time points. CGM profiles were missing from $45 \%$ at either baseline or week 52 visits (Supplementary Table S3). Missing profiles due to site closure and technical issues were considered missing completely at random. Proportions of profiles missing due to withdrawal of subjects were similar across treatment groups, and baseline characteristics of patients in the sub-study with available CGM data at week 52 (Supplementary Table S4) were well matched and similar to those of the full DUAL I extension trial population. ${ }^{15}$

In the DUAL I extension CGM sub-study, mean IG decreased more with IDegLira than with liraglutide (ETD $-1.0 \mathrm{mmol} / \mathrm{L}, 95 \% \mathrm{CI}-1.5$ to $-0.5, P<0.0001)$; IDeg produced a similar reduction to IDegLira (ETD $0.0 \mathrm{mmol} / \mathrm{L}$, $95 \% \mathrm{CI}-0.5$ to $0.5, P=0.9655$ ) (Table 3 ). Statistically significantly lower IG fluctuations (treatment ratio $0.8,95 \%$ CI 0.7 to $0.9, P=0.0018$ ) and a greater reduction in postprandial IG increments across all meals (ETD- $0.5 \mathrm{mmol} / \mathrm{L},-0.9$ to $-0.1, P=0.0288$ ) were observed with IDegLira versus IDeg (Table 3). IG fluctuations and postprandial IG increments were similar with IDegLira versus liraglutide (Table 3). Results were generally consistent across other measures of glycemic fluctuation (MAGE, LBGI, HBGI); however, there were no statistically significant differences between IDegLira and IDeg for LBGI and HBGI (Supplementary Table S5).
From a similar distribution at baseline, more time was spent within IG target range ( 3.9 to $<9.0 \mathrm{mmol} / \mathrm{L}$ ) for all three treatment groups at week 52 (Fig. 2). Time (hours/day) outside IG target range was statistically significantly lower with IDegLira than with liraglutide (ETD-2.2 h/day, 95\% CI -3.8 to $-0.6, P=0.0072$ ), but there was no statistically significant difference in time outside range between IDegLira and IDeg (ETD-0.7 h/day, 95\% CI -2.4 to $1.0, P=0.4310$ ). The duration of IG $<3.9$ and $<3.1 \mathrm{mmol} / \mathrm{L}$ was similar in patients treated with IDegLira compared to liraglutide or IDeg alone. In contrast, the rate of episodes (number per $100 \mathrm{~h}$ ) of IG $<3.9 \mathrm{mmol} / \mathrm{L}$ with IDegLira was statistically significantly lower than with IDeg (treatment rate ratio $0.6,95 \%$ CI 0.4 to $1.0, P=0.0357)$ and higher than with liraglutide (treatment rate ratio $2.2,95 \% \mathrm{CI} 1.3$ to $3.8, P=0.0045$ ). The rate of episodes (number per $100 \mathrm{~h}$ ) of IG $<3.1 \mathrm{mmol} / \mathrm{L}$ was similar for IDegLira compared to liraglutide or IDeg alone (Fig. 2). A similar pattern of results was observed for rates of episodes of low IG $(<3.9$ and $<3.1 \mathrm{mmol} / \mathrm{L})$ during the nocturnal period (00:01-05:59), but low event numbers dictate that meaningful statistical comparisons were not possible for nocturnal episodes (Supplementary Table S6).

Day-to-day IG variability (SD of daily [24 h] mean) and variability of fasting IG (20 min before breakfast) was similar with IDegLira, IDeg, or liraglutide (Table 3).

\section{Discussion}

In this post hoc analysis of two randomized, controlled trials in T2D, data captured via nine-point SMBG profiles and CGM were utilized to more precisely characterize fluctuation

Table 3. Glycemic Fluctuation and Variability in Patients with Type 2 Diabetes Treated for 52 Weeks in the DUAL I Extension Continuous Glucose Monitoring Sub-Study

\begin{tabular}{lccccc}
\hline CGM parameter & $\begin{array}{c}\text { IDegLira } \\
(\mathrm{n}=131)\end{array}$ & $\begin{array}{c}\text { IDeg } \\
(\mathrm{n}=64)\end{array}$ & $\begin{array}{c}\text { ETD [95\% CI]: } \\
\text { IDegLira vs. IDeg }\end{array}$ & $\begin{array}{c}\text { Lira } \\
(\mathrm{n}=65)\end{array}$ & $\begin{array}{c}\text { ETD [95\% CI]: } \\
\text { IDegLira vs. Lira }\end{array}$ \\
\hline $\begin{array}{l}\text { Mean IG (mmol/L) } \\
\text { Mean at baseline }\end{array}$ & $10.2(2.2)$ & $10.2(2.1)$ & & $10.0(2.5)$ & \\
$\quad$ Mean $\Delta$, w52 & $-3.5(1.9)$ & $-3.6(1.9)$ & $\begin{array}{c}0.0[-0.5 \text { to } 0.5] \\
(P=0.9655)\end{array}$ & $-2.5(2.5)$ & $\begin{array}{c}-1.0[-1.5 \text { to }-0.5] \\
(P<0.0001)\end{array}$
\end{tabular}

IG fluctuation (adjusted integrated absolute distance from the mean profile, i.e., flatness of IG profile, $\mathrm{mmol} / \mathrm{L}$ )
Geometric mean at baseline
Geometric mean at w52
$\begin{array}{ll}1.5 & 1.6 \\ 1.0 & 1.3\end{array}$

Ratio: 0.8 [0.7 to 0.9$]$ $(P=0.0018)$
1.5
1.1

Ratio: 1.0 [0.8 to 1.1$]$ $(P=0.6681)$

$1.5(1.2)$

$-0.5[-0.9$ to -0.1$]-0.2(1.4)$ $(P=0.0288)$

$-0.2[-0.6$ to 0.2$]$ $(P=0.3039)$

$0.8(0.1)$

$-0.1[-0.2$ to 0.1$] \quad 0.5(0.1)$ $(P=0.4197)$

$0.1[-0.0$ to 0.3$]$

$(P=0.1056)$

Day-to-day variability of fasting IG, 20 min before breakfast (SD of daily fasting IG, mmol/L) Geometric mean at baseline (CV) 0.8 (115.4) 0.7 (130.4) Geometric mean at w52 (CV)

0.6 (106.7) 0.5 (139.9) Ratio: 0.9 [0.6 to 1.3$]$ $(P=0.4811)$
$0.8(127.5)$

0.5 (121.1) Ratio: 0.8 [0.5 to 1.2$]$ $(P=0.2462)$

Values are mean (SD) unless otherwise stated. CGM parameters are analyzed based on observed data using an ANCOVA method with treatment, region, baseline $\mathrm{HbA}_{1 \mathrm{c}}$ stratum $(\leq 8.3 \%[\leq 67 \mathrm{mmol} / \mathrm{mol}],>8.3 \%$ [ $\left.>67 \mathrm{mmol} / \mathrm{mol}]\right)$, and previous OAD treatment as fixed effects. Baseline values of parameters were included as covariates for mean IG and postprandial IG increment. Fluctuation is log-transformed before analysis.

ANCOVA, analysis of covariance; CV, coefficient of variation; ETD, estimated treatment difference; IG, interstitial glucose; Lira, liraglutide; SE, standard error; w, week. 


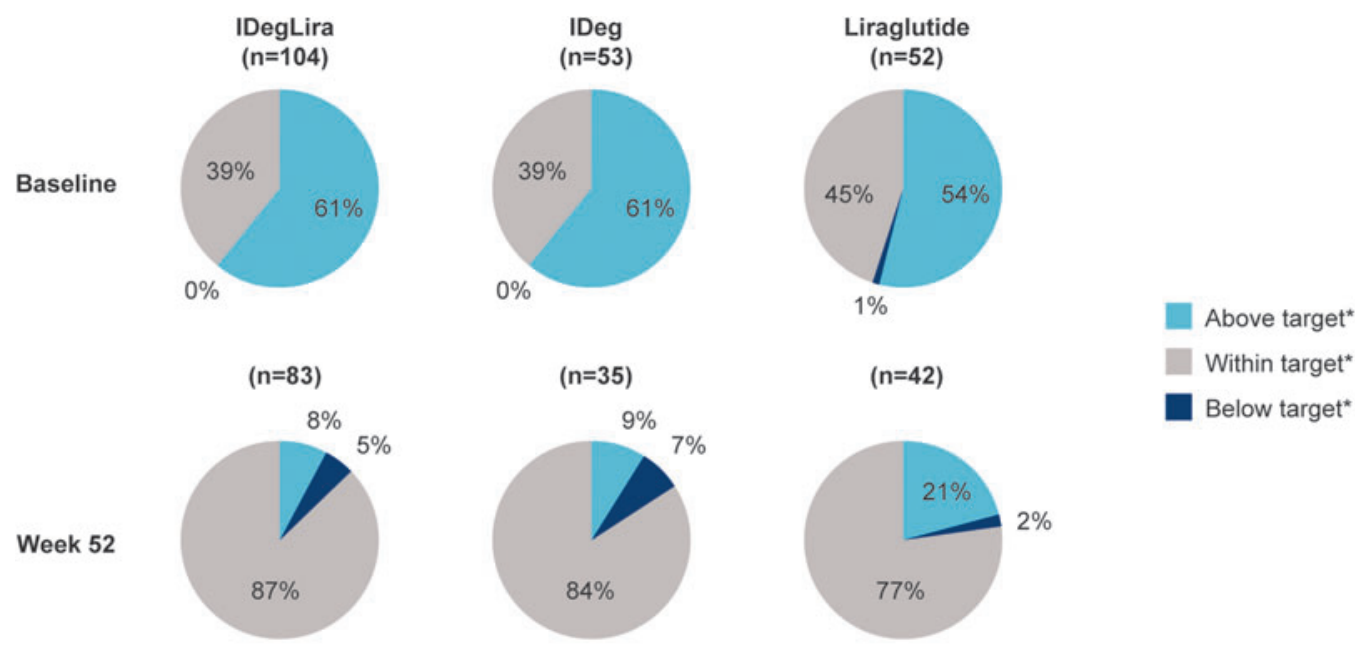

\begin{tabular}{|c|c|c|c|c|c|}
\hline & IDegLira & IDeg & $\begin{array}{c}\text { ETD [95\% CI]: } \\
\text { IDegLira vs. IDeg }\end{array}$ & Lira & $\begin{array}{l}\text { ETD [95\% Cl]: } \\
\text { IDegLira vs. Lira }\end{array}$ \\
\hline $\begin{array}{l}\text { Time outside IG target range, hours/day } \\
\text { Mean at baseline (SD) } \\
\text { Mean at week } 52 \text { (SD) }\end{array}$ & $\begin{array}{c}14.7(7.1) \\
3.2(3.8)\end{array}$ & $\begin{array}{c}14.6(6.7) \\
3.8(2.6)\end{array}$ & $\begin{array}{c}-0.7[-2.4,1.0] \\
p=0.4310\end{array}$ & $\begin{array}{c}13.2(7.2) \\
5.6(6.2)\end{array}$ & $\begin{array}{c}-2.2[-3.8,-0.6] \\
p=0.0072\end{array}$ \\
\hline $\begin{array}{l}\text { IG }<3.9 \mathrm{mmol} / \mathrm{L} \\
\text { Duration, hours/day } \\
\text { Mean at baseline (SD) } \\
\text { Mean at week } 52(\mathrm{SD}) \\
\\
\text { Episodes, number/100 hours } \\
\text { Rate at baseline } \\
\text { Rate at week } 52\end{array}$ & $\begin{array}{l}0.1(0.4) \\
1.2(2.3) \\
\\
\\
0.6 \\
3.7\end{array}$ & $\begin{array}{l}0.1(0.3) \\
1.7(1.8) \\
\\
\\
0.6 \\
6.1\end{array}$ & $\begin{array}{c}-0.4[-1.2,0.5] \\
\quad p=0.3921 \\
\text { Ratio: } 0.6[0.4,1.0] \\
p=0.0357\end{array}$ & $\begin{array}{c}0.2(0.9) \\
0.5(2.1) \\
\\
\\
0.5 \\
1.9\end{array}$ & $\begin{array}{c}0.7[-0.0,1.5] \\
p=0.0582 \\
\text { Ratio: } 2.2[1.3,3.8] \\
p=0.0045\end{array}$ \\
\hline $\begin{array}{l}\text { IG }<3.1 \mathrm{mmol} / \mathrm{L} \\
\text { Duration, hours/day } \\
\text { Mean at baseline (SD) } \\
\text { Mean at week } 52(\mathrm{SD}) \\
\\
\text { Episodes, number/100 hours } \\
\text { Rate at baseline } \\
\text { Rate at week } 52\end{array}$ & $\begin{array}{c}0.1(0.3) \\
0.4(1.1) \\
\\
0.3 \\
1.3\end{array}$ & $\begin{array}{c}0.0(0.2) \\
0.4(0.6) \\
\\
\\
0.4 \\
2.0\end{array}$ & $\begin{array}{c}\text { Ratio: } 0.7[0.3,1.5] \\
p=0.3354\end{array}$ & $\begin{array}{c}0.1(0.7) \\
0.2(0.8) \\
\\
0.4 \\
0.6\end{array}$ & $\begin{array}{c}\text { Ratio: } 1.9[0.8,4.5] \\
p=0.1537\end{array}$ \\
\hline
\end{tabular}

FIG. 2. Time (\%) above ( $\mathrm{IG} \geq 9.0 \mathrm{mmol} / \mathrm{L})$, within $(3.9 \leq \mathrm{IG}<9.0 \mathrm{mmol} / \mathrm{L})$ and below ( $\mathrm{IG}<3.9 \mathrm{mmol} / \mathrm{L}) \mathrm{IG}$ target range* for patients in the DUAL I extension CGM sub-study. Sub-study analysis set. *IG target range: 3.9 to $<9.0 \mathrm{mmol} / \mathrm{L}$. Analysis is based on observed data using an ANCOVA method for time/duration, and a negative binomial regression model for episodes (using a log link and the logarithm of the profile duration as offset) with treatment, region, baseline $\mathrm{HbA}_{1 \mathrm{c}}$ stratum $(\leq 8.3 \%$ [ $\leq 67 \mathrm{mmol} / \mathrm{mol}],>8.3 \%$ [ $>67 \mathrm{mmol} / \mathrm{mol}]$ ) and previous OAD treatment as fixed effects for both methods. CGM, continuous glucose monitoring; CI, confidence interval; IG, interstitial glucose; Lira, liraglutide; SD, standard deviation.

in daily blood glucose values, thereby allowing a more indepth evaluation of diabetes treatment. These new data support the main trial findings and provide further evidence that the complementary effects of IDeg and liraglutide allow more patients treated with IDegLira to achieve their glycemic target.

The analysis of DUAL I extension CGM data showed that treatment with IDegLira resulted in a greater reduction of mean IG compared to liraglutide, but not compared to IDeg. This is consistent with the previously reported finding that IDegLira reduced the mean nine-point profile significantly more than liraglutide, but conferred a similar reduction to IDeg. ${ }^{15}$ The present analysis of the nine-point profiles also showed that treatment with IDegLira results in more patients achieving preprandial targets than with IDeg in DUAL II, at the exact same dose of IDeg. This indicates the contribution of the lir- aglutide component in reducing preprandial glucose values. The same result was also observed in our analysis of DUAL I, although at a lower dose of IDeg in the IDegLira arm. The effects of the IDeg component of IDegLira on fasting glucose levels results in a shifting of the glucose profile downward compared to liraglutide alone, but liraglutide also contributes the overall preprandial glucose reduction with IDegLira.

The glucose-dependent effects of liraglutide on postprandial glucose are maintained in IDegLira and translate to a flattening of the blood glucose curve compared to IDeg alone. Although not statistically significant, CGM data suggest that IDegLira resulted in greater reductions in overall IG fluctuation, and postprandial IG increment across all meals, compared with IDeg. IDegLira led to a significantly greater reduction in SMBG range over $24 \mathrm{~h}$ than IDeg in both trials. This is partly explained by higher endogenous insulin 
secretion and improved beta cell function with IDegLira. ${ }^{22}$ These results are consistent with, and extend the primary results from, the DUAL I extension and DUAL II in which IDegLira resulted in a lower mean EOT postprandial glucose increment after all main meals for IDegLira versus IDeg. ${ }^{13,15}$

This analysis further confirmed that the combined effects of IDegLira to lower (versus liraglutide) and flatten (versus IDeg) the blood glucose curve translated into a larger proportion of patients having SMBG values within the defined target range (see Materials and Methods section) compared to IDeg or liraglutide alone. This applied to the analyses of all three postprandial values, all four preprandial values, and the entire nine-point SMBG profile. Similarly, the CGM results demonstrate that the time spent outside range with IDegLira was less than with liraglutide. Although the duration of IG $<3.9$ and $<3.1 \mathrm{mmol} / \mathrm{L}$ was not statistically significantly different for IDegLira and liraglutide or IDeg, the rate of IG $<3.9 \mathrm{mmol} / \mathrm{L}$ with IDegLira was lower than with IDeg and higher than with liraglutide. This latter finding is consistent with the main DUAL I trial, ${ }^{14,15}$ which assessed episodes (rate) of hypoglycemia rather than duration. Furthermore, while interpretation of results for episodes of low IG during the nocturnal period should be cautious given the low number of episodes, a higher rate of episodes with IDegLira than with liraglutide is also consistent with the results for nocturnal hypoglycemia from the main DUAL I trial. ${ }^{15}$

The properties of IDeg with respect to day-to-day variability are maintained in IDegLira; day-to-day variabilitybased on CGM data-was similar with all three treatments. The data from DUAL I extension (assessed based on prebreakfast SMBG and CGM data, and mean IG from CGM) showed no significant differences between IDegLira and IDeg, and although the prebreakfast SMBG data showed statistically significant greater variability with IDegLira than liraglutide, this was not confirmed by the CGM data. In the DUAL II trial the prebreakfast SMBG data indicated statistically significantly lower day-to-day variability with IDegLira than with IDeg. Care should be exercised in the clinical interpretation of these data due to the inconsistency of these results between the two trials.

Although there is debate regarding whether reducing postprandial glucose is more important than reducing preprandial glucose levels, lowering both is clearly essential if patients are to achieve $\mathrm{HbA}_{1 \mathrm{c}}$ targets. ${ }^{23}$ Due to the complementary action of its components IDeg and the GLP-1 receptor agonist liraglutide, IDegLira allows patients with T2D to address both fasting and postprandial glucose levels. Lowering overall $\mathrm{HbA}_{1 \mathrm{c}}$ may not be a sufficient indicator of optimal glycemic control for every patient, and it is increasingly recognized that reductions in $\mathrm{HbA}_{1 \mathrm{c}}$ should also be accompanied by reduction in glycemic fluctuations. Indeed, large fluctuations can be masked by a "good" $\mathrm{HbA}_{1 \mathrm{c}}$ level, and if present, they may contribute to increased risk of microvascular and metabolic complications, postmeal fatigue, feelings of poor well-being, and other adverse patient-reported outcomes associated with diabetes treatments. ${ }^{10,11,26-29}$ Measures of glucose fluctuation together with patient-reported outcomes and focus group interviews may allow improved outcomes and potentially greater longterm adherence to medications.

When considering the limitations of this study, it is important to remember that this was a post hoc analysis of data generated in a clinical trial setting for a defined time period (up to 52 weeks), with a more homogeneous population having more regular consultations than are possible in real world clinical practice. In the DUAL II trial, dosing of IDeg was limited to a maximum of $50 \mathrm{U}$ to balance insulin exposure between arms and isolate the contribution of the liraglutide component of IDegLira. However, this dose restriction also limits comparisons between IDeg treatment in DUAL II and in real life. Also, nine-point SMBG measures are a relatively imprecise measure of glucose fluctuations compared to CGM. In the case of the CGM data, withdrawal of subjects, site closure, and technical issues also meant that there was a substantial proportion of CGM data missing. The demographics of patients with available data were similar to the sub-study population (FAS), but there remains the possibility that a full dataset would show different results. CGM data were not recorded in the DUAL II study, ${ }^{13}$ and comparison across the trials is therefore not possible for the CGM endpoints. CGM technology is rapidly improving, and although this study had higher rates of failed sensor placement and incomplete readings than desired, it still provides a valuable framework for measuring fluctuation. The CGM findings also correlated well with the initial glucose management outcomes from the DUAL I extension and DUAL II parent trials. ${ }^{13,15}$

From a clinical perspective, the results of this post hoc analysis are relevant and important for both the patient and the treating physician. First and foremost, they further refine our understanding of how and why the additional $\mathrm{HbA}_{1 \mathrm{c}^{-}}$ lowering benefits of IDegLira over IDeg or liraglutide alone can be realized without increasing the risk of hypoglycemia, compared with IDeg. This lowered risk is particularly important in real world practice, where avoidance of hypoglycemia is a significant safety concern for the patient, carers, and medical providers. ${ }^{24,25}$ Reduced hypoglycemia may also allow patients to titrate medication to achieve lower blood glucose targets.

In conclusion, the results from these secondary analyses, using two different methodologies to assess BG fluctuations, consistently support the main results from the parent trials demonstrating greater improvements in glycemic control with IDegLira versus lira alone ${ }^{15}$ or IDeg alone. ${ }^{14,15}$ These new findings on glycemic fluctuation provide additional clarification about how the complementary modes of action of IDeg and liraglutide can help more patients achieve glycemic targets. IDegLira shifted the blood glucose profile downward compared to liraglutide, flattened the profile compared to IDeg, and was associated with reduced glycemic fluctuations compared to the individual components alone, allowing more patients to achieve their average blood glucose targets while remaining within target levels for a higher proportion of the day.

\section{Acknowledgments}

The authors thank Keval Chandarana of Novo Nordisk A/ $\mathrm{S}$ for review and input to the article, and Charlie Hunt and Beverly La Ferla at Watermeadow Medical (funded by Novo Nordisk), for medical writing and editorial assistance. This analysis, and the DUAL I extension (ClinicalTrials.gov number: NCT01336023) and DUAL II (ClinicalTrials.gov number: NCT01392573) trials were funded by Novo Nordisk A/S. 


\section{Author Disclosure Statement}

A.B.K. has received research support from Sanofi, Lilly, Novo Nordisk, Medtronic, Animas, Takeda, and Amylin, speaker fees from Sanofi, Lilly, Novo Nordisk, Medtronic, Animas, Takeda, Janssen, Lifescan, Pfizer, GlaxoSmithKline, and Amylin, and he has acted as a consultant to Sanofi, Lilly, Novo Nordisk, Medtronic, Animas, Takeda, Janssen, and Lifescan. A.P.-T. has served on behalf of her organization on advisory panels for Eli Lilly, Novo Nordisk, Merck and Co., and Sanofi-Aventis, and she has received research support from Mylan, Lexicon, DexCom, Novo Nordisk, Sanofi-Aventis, Eli Lilly and Company, and AstraZeneca LP. AP-T does not receive any personal reimbursement for these functions. E.S.K. has served on advisory panels for Novo Nordisk and Pfizer. K.B. and I.H.L. are fulltime employees of, and own stock at, Novo Nordisk A/S. T.V. has received lecture fees from Amgen, AstraZeneca, Boehringer Ingelheim Pharmaceuticals, Bristol-Myers Squibb, Eli Lilly and Company, Merck Sharp \& Dohme, Novo Nordisk, Novartis, Sanofi, and Zealand Pharma, and has been an advisory-board member for Amgen, Janssen, Novo Nordisk, Merck Sharp \& Dohme, Takeda, and Bristol-Myers Squibb/ AstraZeneca.

\section{Contributor Statements}

A.P.-T. and T.V. were trial investigators and contributed significantly to the conduct of the study and acquisition of clinical data. K.B. performed the statistical analyses. All authors were involved in review and interpretation of the data, preparing the first draft of the article, and critical revision for important intellectual content. All authors approved the final version of the article and take full responsibility for the content.

\section{References}

1. Inzucchi SE, Bergenstal RM, Buse JB, et al.: Management of hyperglycemia in type 2 diabetes, 2015: a patient-centered approach: update to a position statement of the American Diabetes Association and the European Association for the Study of Diabetes. Diabetes Care 2015;38:140-149.

2. Zenari L, Marangoni A: What are the preferred strategies for control of glycaemic variability in patients with type 2 diabetes mellitus? Diabetes Obes Metab 2013;15(Suppl. 2):17-25.

3. Chon S, Lee YJ, Fraterrigo G, et al.: Evaluation of glycemic variability in well-controlled type 2 diabetes mellitus. Diabetes Technol Ther 2013;15:455-460.

4. International Diabetes Federation: 2011 Guideline for the Management of PostMeal Glucose in Diabetes. Brussels: IDF; 2011. http://www.idf.org/sites/default/files/postmeal\% 20glucose\%20guidelines.pdf (accessed March 6 2017)

5. Nalysnyk L, Hernandez-Medina M, Krishnarajah G: Glycaemic variability and complications in patients with diabetes mellitus: evidence from a systematic review of the literature. Diabetes Obes Metab 2010;12:288-298.

6. Qu Y, Jacober SJ, Zhang Q, et al.: Rate of hypoglycemia in insulin-treated patients with type 2 diabetes can be predicted from glycemic variability data. Diabetes Technol Ther 2012;14:1008-1012.

7. Cavalot F, Petrelli A, Traversa M, et al.: Postprandial blood glucose is a stronger predictor of cardiovascular events than fasting blood glucose in type 2 diabetes mellitus, particu- larly in women: lessons from the San Luigi Gonzaga Diabetes Study. J Clin Endocrinol Metab 2006;91:813-819.

8. DECODE Study Group. Glucose tolerance and cardiovascular mortality: comparison of fasting and 2-hour diagnostic criteria. Arch Intern Med 2001;161:397-405.

9. Nakagami T, Qiao Q, Tuomilehto J, et al.: Screen-detected diabetes, hypertension and hypercholesterolemia as predictors of cardiovascular mortality in five populations of Asian origin: the DECODA study. Eur J Cardiovasc Prev Rehabil 2006;13:555-561.

10. Hirsch IB: Glycemic variability and diabetes complications: does it matter? Of course it does! Diabetes Care 2015;38: 1610-1614.

11. Bergenstal RM: Glycemic variability and diabetes complications: does it matter? Simply put, there are better glycemic markers! Diabetes Care 2015;38:1615-1621.

12. Eng C, Kramer CK, Zinman B, Retnakaran R: Glucagonlike peptide-1 receptor agonist and basal insulin combination treatment for the management of type 2 diabetes: a systematic review and meta-analysis. Lancet 2014;384: 2228-2234.

13. Buse JB, Vilsboll T, Thurman J, et al.: Contribution of liraglutide in the fixed-ratio combination of insulin degludec and liraglutide (IDegLira). Diabetes Care 2014;37: 2926-2933.

14. Gough SC, Bode B, Woo V, et al.: Efficacy and safety of a fixed-ratio combination of insulin degludec and liraglutide (IDegLira) compared with its components given alone: results of a phase 3, open-label, randomised, 26-week, treat-to-target trial in insulin-naive patients with type 2 diabetes. Lancet Diabetes Endocrinol 2014;2:885-893.

15. Gough SC, Bode BW, Woo VC, et al.: One-year efficacy and safety of a fixed combination of insulin degludec and liraglutide in patients with type 2 diabetes: results of a 26week extension to a 26-week main trial. Diabetes Obes Metab 2015;17:965-973.

16. Zinman B, Philis-Tsimikas A, Cariou B, et al.: Insulin degludec versus insulin glargine in insulin-naive patients with type 2 diabetes: a 1-year, randomized, treat-to-target trial (BEGIN Once Long). Diabetes Care 2012;35:2464 2471.

17. Buse JB, Rosenstock J, Sesti G, et al.: Liraglutide once a day versus exenatide twice a day for type 2 diabetes: a 26week randomised, parallel-group, multinational, open-label trial (LEAD-6). Lancet 2009;374:39-47.

18. World Medical Association. Declaration of Helsinki: Ethical Principles for Medical Research Involving Human Subjects. Last amended by the 59th WMA Assembly Seoul, October, 2008.

19. International Conference on Harmonisation Working Group. ICH Harmonised Tripartite Guideline: Guideline for Good Clinical Practice E6(R1). Geneva International Conference on Harmonisation of Technical Requirements for Registration of Pharmaceuticals for Human Use. June 10, 1996. https://www.ich.org/fileadmin/Public_Web_Site/ ICH_Products/Guidelines/Efficacy/E6/E6_R1_Guideline.pdf (accessed March 6, 2017).

20. Daenen S, Sola-Gazagnes A, M'Bemba J, et al.: Peak-time determination of post-meal glucose excursions in insulintreated diabetic patients. Diabetes Metab 2010;36:165-169.

21. American Diabetes Association: Standards of medical care in diabetes, 2014. Diabetes Care 2014;37:S14-S80.

22. Holst JJ, Buse JB, Rodbard HW, et al.: IDegLira improves both fasting and postprandial glucose control as 
demonstrated using continuous glucose monitoring and a standardized meal test. J Diabetes Sci Technol 2015;10: 389-397.

23. Ceriello A, Kilpatrick ES: Glycemic variability: both sides of the story. Diabetes Care 2013;36:S272-S275.

24. Graveling AJ, Frier BM: Hypoglycaemia: an overview. Primary Care Diabetes 2009;3:131-139.

25. Williams SA, Pollack MF, DiBonaventura M: Effects of hypoglycemia on health-related quality of life, treatment satisfaction and healthcare resource utilization in patients with type 2 diabetes mellitus. Diabetes Res Clin Pract 2011; 91:363-370.

26. Fritschi C, Quinn L: Fatigue in patients with diabetes: a review. J Psychosom Res 2010;69:33-41.

27. Penckofer S, Quinn L, Byrn M, et al.: Does glycemic variability impact mood and quality of life? Diabetes Technol Ther 2012;14:303-310.
28. Peyrot M, Rubin RR, Chen X, Frias JP: Associations between improved glucose control and patient-reported outcomes after initiation of insulin pump therapy in patients with type 2 diabetes mellitus. Diabetes Technol Ther 2011; 13:471-476.

29. Ayano-Takahara S, Ikeda K, Fujimoto S, et al.: Glycemic variability is associated with quality of life and treatment satisfaction in patients with type 1 diabetes. Diabetes Care 2015;38:e1-e2.

Address correspondence to: Allen B. King, MD

Diabetes Care Center

1260 S. Main Street \#201

Salinas, CA 93901

E-mail: aking@diabetescarecenter.com 